\title{
Apparent rotation in three-dimensional space: Effects of temporal, spatial, and structural factors
}

\author{
JAMES T. TODD, ROBIN A. AKERSTROM, and FRANCENE D. REICHEL \\ Brandeis University, Waltham, Massachusetts \\ and \\ WILLIAM HAYES \\ Albion College, Albion, Michigan
}

\begin{abstract}
Several experiments were performed in an effort to determine the different combinations of display parameters that can reliably elicit a perceptually compelling impression of a solid object rotating rigidly in three-dimensional space. All of the displays were computer-generated simulations of rigid objects rotating in depth under parallel projection. The parameters investigated included the number of frames in the apparent motion sequence, the temporal and spatial displacements between frames, the number of elements in the depicted object, and the structural organization of those elements. The results indicated that there are large interactions among these different parameters in their effects on the perception of structure from motion.
\end{abstract}

In a classic series of experiments performed over three decades ago, Wallach and O'Connell (1953) demonstrated that the pattern of optical distortion produced by a moving object provides perceptually compelling information about its 3-D structure. Numerous computational analyses have been reported in the literature in an effort to provide a theoretical explanation of this phenomenon. Some of these analyses have been primarily concerned with smoothly varying patterns of optical flow, such as those produced when an observer locomotes over a rigid ground surface (Clocksin, 1980; Koenderink, 1986; Koenderink \& van Doorn, 1975, 1977; Lee, 1974; LonguetHiggens \& Prazdny, 1980; Nakayama \& Loomis, 1974; Prazdny, 1980), whereas others have examined more general configurations of moving objects, such as wireframe figures rotating in depth (Hoffman \& Bennett, 1985, 1986; Todd, 1982; Ullman, 1979, 1984; Webb \& Aggarwal, 1981).

A considerable amount of psychological research has been designed to establish the psychological validity of these analyses (Braunstein \& Andersen, 1984; Braunstein, Hoffman, Shapiro, Andersen, \& Bennett, 1987; Doner, Lappin, \& Perfetto, 1984; Lappin, Doner, \& Kottas, 1980; Lappin \& Fuqua, 1983; Todd, 1981, 1982, 1984, 1985). Much of this research has focused specifically on the theoretical limits suggested by different models on the minimal number of points and/or views of an apparent motion sequence required, in principle, to provide reli-

This research was supported by the National Science Foundation (Grant BNS-8420143), the Office of Naval Research, and the Air Force Office of Scientific Research (\#F49620-87-C-0018). We wish to thank John Andersen, Mike Braunstein, and Joe Lappin for their helpful suggestions at various stages of this project. Requests for reprints should be addressed to James T. Todd, Department of Psychology, Brandeis University, Waltham, MA 02254. able information about an object's 3-D structure. The basic idea has been to compare these theoretical limits with the perceptual capabilities of actual human observers.

It is important to keep in mind that even when a display is computationally sufficient to determine an object's 3-D structure, an observer may be unable to perceive that structure because of spatial, temporal, or structural limitations of the human visual system. This is particularly true for apparent motion sequences under minimal conditions. If the individual views of an object are presented too briefly, or its spatial displacements are too large, or it is structurally too complex, a human observer may be unable to cope with available information that would be adequate, in principle, for an ideal observer.

To what extent do these biological limitations arise in current research on the perception of structure from motion? There are surprisingly few data to answer this question with any degree of confidence. Although there is a vast literature on the perception of apparent motion, it has been largely restricted to two-frame sequences depicting simple translations in 2-D space. There is some evidence to suggest that the results for this type of display may not generalize to other conditions. Within the domain of 2-D motion, for example, Sperling (1976) observed that the optimal timing and displacement parameters for two-frame sequences may differ by an order of magnitude from those that are appropriate for multiple-frame sequences. A similar result was also obtained for the perception of motion in depth. Shepard and Judd (1976) reported that the perception of rigid rotation from a two-frame apparent motion sequence in oscillation requires a minimal stimulus onset asynchrony (SOA) of several hundred milliseconds-a value that is many times greater than that typically employed with longer length sequences (e.g., in cinematography). 
The present series of experiments was designed to examine the biological limits on the perception of structure from motion over a much broader range of conditions than has been considered in previous investigations. All of the displays employed in these experiments were computergenerated simulations of rigid objects rotating in depth under parallel projection. The goal of the research was to determine empirically the different combinations of display parameters that can reliably elicit a perceptually compelling impression of rigid rotation in 3-D space. The parameters investigated included the number of frames in the apparent motion sequence, the temporal and spatial displacements between frames, the number of elements in the depicted object, and the structural organization of those elements.

\section{GENERAL METHOD}

\section{Apparatus}

Stimuli were produced using a Lex-90 graphics system and were displayed on a 19-in. color monitor. When the red, green, and blue phosphors of this monitor were illuminated together at maximum intensity, they decayed by $97 \%$ within $2 \mathrm{msec}$. The approximate viewing distance was $115 \mathrm{~cm}$; there were no restrictions on observers' head or body movements. The stimuli were presented within a rectangular region of the display screen that was $33 \mathrm{~cm}$ along the horizontal axis and $26 \mathrm{~cm}$ along the vertical axis. The spatial resolution within this viewing window was $320 \times 256$ pixels. Thus, each pixel had a horizontal and vertical extent of approximately $0.1 \mathrm{~cm}$, producing a visual angle of approximately $3^{\prime}$.

To generate a moving display, up to nine separate images could be loaded simultaneously into the graphics frame buffer. The effect of motion was obtained by presenting these images in rapid succession, cycling back and forth through the sequence at a fixed rate. The motion sequence was controlled directly by the graphics processor so that each shift from one image to the next always occurred within the vertical retrace period of the raster display cycle. The refresh rate of the system was $60 \mathrm{~Hz}$. Thus, the minimal SOA that could be achieved was $16.7 \mathrm{msec}$. The interstimulus interval (ISI) could also be manipulated by inserting blank frames into the sequence, but the total number of frames, including blanks, could not exceed nine.

\section{Stimuli}

The stimuli were created from computer simulations of wire-frame figures rotating in depth under parallel projection. The specific configuration of line segments used in the simulation was generated at random on every trial. Each line segment had one of its endpoints randomly positioned in 3-D space at a distance of between 80 and 120 pixels from the origin at the center of the display screen. Three of the line segments in each display had their other endpoints positioned at the origin. All additional lines were connected to the endpoints of other segments. In most of our experiments, the rotating figures were composed of only 4 line segments, although in Experiments 6 and 7, the number of line segments was systematically varied. (Examples of configurations with 4 and 64 line segments are shown in the upper panels of Figure 1.)

Between the frames of a motion sequence, the randomly generated configuration of line segments was rotated in 3-D space by a fixed angular displacement of $6^{\circ}, 18^{\circ}$, or $30^{\circ}$ about a vertical axis. It is important to keep in mind that there is only a probabilistic relationship between the rotation of a point in 3-D space and its corresponding displacement on the display screen. The projected displacement of a point varies with phase from 0 to $d \sin (\theta / 2)$, where $\theta$ is the angular displacement of the point in 3-D space and $d$ is the diameter of its circular trajectory. Figure 2 shows the proba-
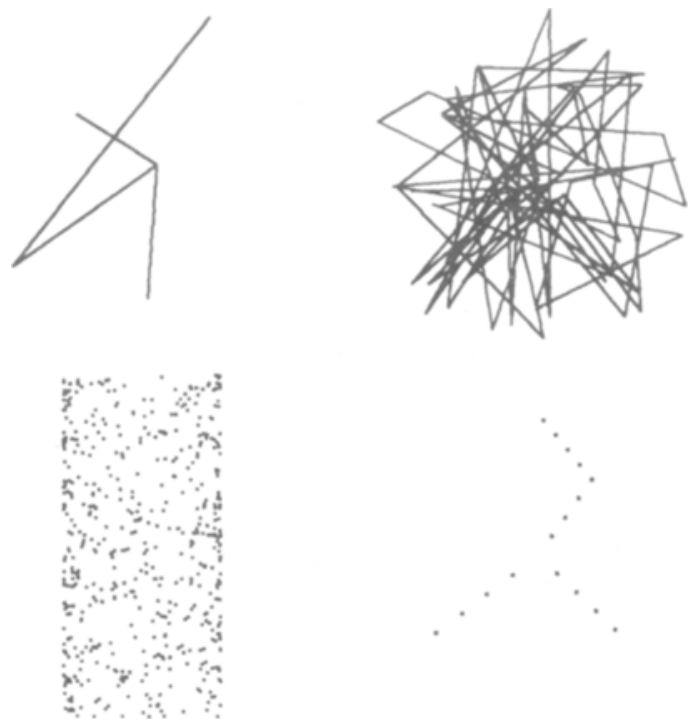

Figure 1. Examples of stimulus configurations used in the present experiments. Clockwise from upper left are a 4-line configuration from Experiments 1-5, a 64-line configuration from Experiments 6 and 7, a 4-line configuration with 4 dots per line from Experiments 8 and 9, and a 500-dot cylindrical surface from Experiments 10 and 11.

bility density functions of projected displacements for the different rotation angles $\left(6^{\circ}, 18^{\circ}\right.$, and $\left.30^{\circ}\right)$ and spatial configurations used in our experiments. Each of these curves was generated empirically from a sample of $1,200,000$ displacements.

The number of frames in a display sequence and their timing were varied in the different experiments. A 0 -msec ISI was used in all cases, except in Experiment 5, in which ISI was systematically manipulated for two-frame sequences. In several other experiments the SOAs were varied between 33 and $800 \mathrm{msec}$, and the number of distinct frames in a sequence was varied between two and nine for a wide range of accompanying conditions.

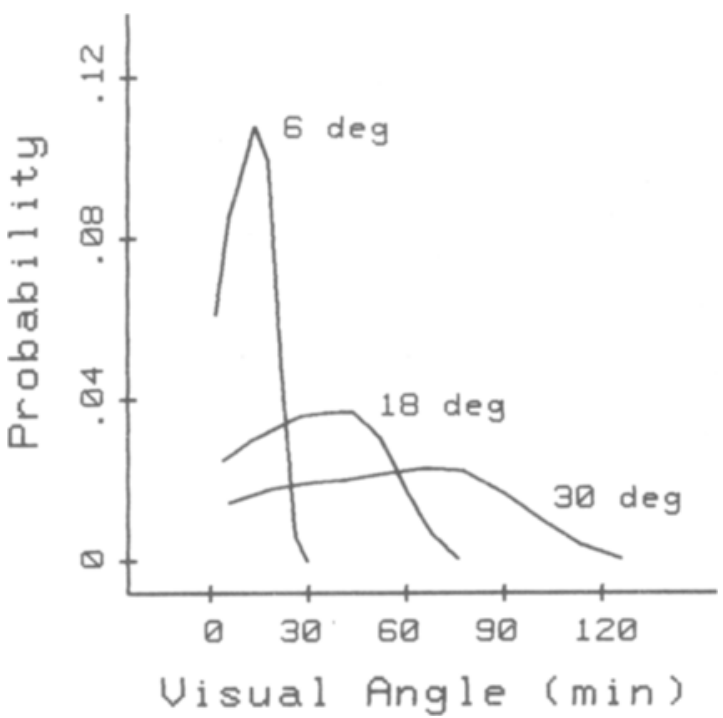

Figure 2. The probability density distributions of projected displacements that were produced when the structural configurations used in the present experiments were rotated in 3-D space with angular displacements of $6^{\circ}, 18^{\circ}$, and $30^{\circ}$. Each curve was generated empirically from a sample of $1,200,000$ displacements. 


\section{Procedure}

The observers' task in all of the experiments was to rate each display on a 10-point rigidity scale by hitting the appropriate key $(0-9)$ on the computer keyboard. The maximum viewing period for any given display was $20 \mathrm{sec}$, although a new trial was never initiated until an appropriate response was recorded. In most instances, observers did not use the full 20 -sec viewing period to select their ratings.

The phenomenological impression produced by these displays is dependent on several variables. Under optimal conditions, a display will be perceived as a 3-D object rotating rigidly in depth. As the parameters of motion depart from their optimal values, the impression of rigid rotation breaks down in stages. With slight deviations from the optimal parameter values, the impression of rotation in depth is retained, but it does not appear to be perfectly rigid. With more drastic deviations, the impression of motion is retained, but it appears to be confined to the 2-D plane of the display screen. Finally, the impression of motion can be eliminated altogether, in which case the display will be perceived as a flickering static form or as a chaotic succession of unrelated forms.

The 10-point rating scale employed in our experiments was designed to reflect the overall pattern of these phenomenological impressions (cf. Shepard \& Judd, 1976). For example, if a display was perceived as a perfectly rigid rotation in depth, it would be given the highest possible rating of 9 . If it appeared to be rotating in depth with a moderate amount of nonrigid distortion, then the rating would be lowered to, say, 6 or 7 . If it appeared as an ambiguous combination of 2- and 3-D motion, then the rating would be lowered still further, to, say, 3 or 4 . If there was a complete absence of perceived motion in depth (including the case of no motion at all), then the display would be given the lowest possible rating of 0 .

Each of the reported experiments was divided into three blocks of 75 trials. The first block in each experiment was intended primarily to familiarize the observer with the overall range of displays in a given set of conditions, and was excluded from subsequent analyses.

\section{Observers}

Ratings were provided by all 4 authors over a period of several months. Each observer viewed several thousand displays during the course of the investigation.

\section{EXPERIMENTS 1 AND 2}

Experiments 1 and 2 were designed to determine how changes in SOA influence the impression of rotation in depth for nine- and two-frame motion sequences.

\section{Method}

Displays were presented with frame-to-frame angular displacements of $6^{\circ}, 18^{\circ}$, or $30^{\circ}$, and with SOAs of 50,100,200,400, or $800 \mathrm{msec}$. Each possible combination of angular displacement and SOA occurred five times during each block of trials. Every display contained 4 line segments, and the individual frames of a sequence were presented with a 0 -msec ISI. Nine-frame sequences were employed in Experiment 1, and two-frame sequences were employed in Experiment 2.

\section{Results}

The data for the nine-frame sequences are presented in Figure 3. The impression of rigid rotation for these sequences was greatest with the smallest possible angular displacement of $6^{\circ}$ and the smallest possible SOA of

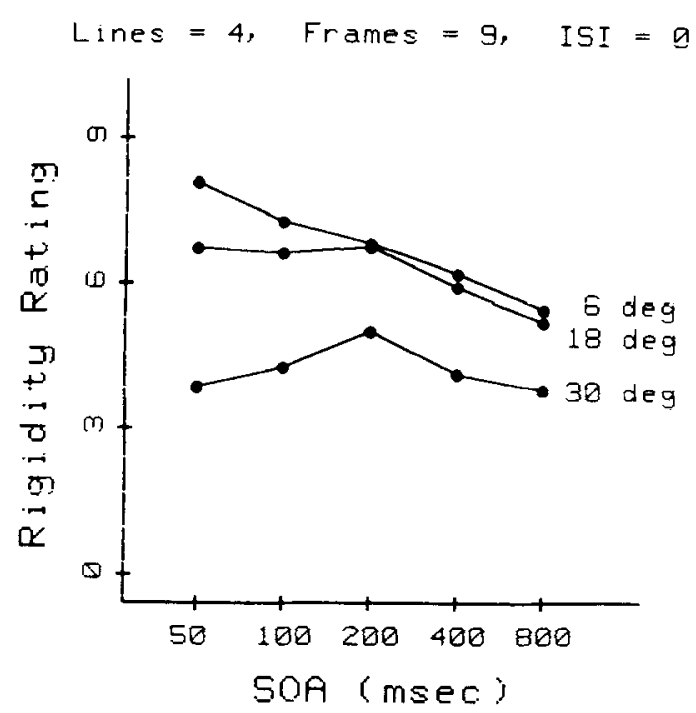

Figure 3. Mean rigidity ratings of 4 observers as a function of SOA for the nine-frame sequences in Experiment 1, with $6^{\circ}, 18^{\circ}$, and $30^{\circ}$ angular displacements.

$50 \mathrm{msec}$. The average rating in that case was 8.1 , indicating that the perception of nonrigid motion was negligible for most of the displays. The ratings decreased sharply with larger angular displacements $[F(2,6)=42.7$, $p<.001]$, and, to a lesser extent, with larger SOAs $[F(4,12)=3.32, p<.05]$.

The two-frame sequences produced a very different pattern of results. As shown in Figure 4, the ratings in that case were affected only slightly by the variations in angular displacement $[F(2,6)=19.54, p<.005]$, and were increased dramatically by larger SOAs $[F(4,12)=94.65$,

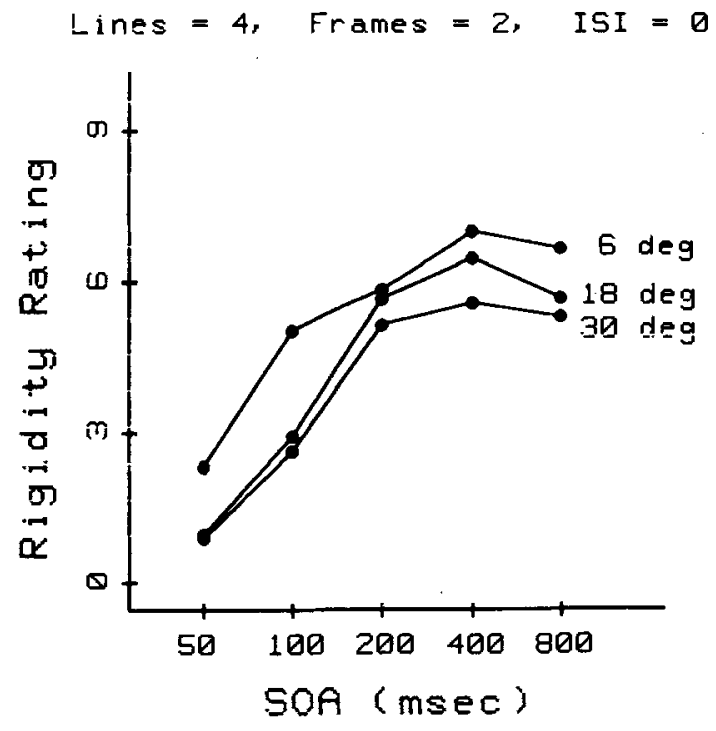

Figure 4. Mean rigidity ratings of 4 observers as a function of SOA for the two-frame sequences in Experiment 2, with $6^{\circ}, 18^{\circ}$, and $30^{\circ}$ angular displacements. 
$p<.001$ ]. Indeed, at an SOA of $50 \mathrm{msec}$, which was optimal for perceiving rigid rotation in a nine-frame sequence, a two-frame sequence appeared as an incoherent pattern of flickering lines in the plane of the display screen.

Another important difference between the two- and nine-frame displays was noted by all 4 observers. Under optimal conditions, with the nine-frame displays, the perception of rigid motion was almost immediate, with no effort on the part of the observer. This was not the case with the two-frame sequences. Even under optimal conditions the impression of rigid motion could take several seconds. Often there was a conscious attempt to mentally rotate the object or to mentally invert some of its line segments in depth so that they all appeared to rotate in the same direction. Nevertheless, once the impression of rigid rotation was achieved, it was quite striking.

\section{EXPERIMENTS 3 AND 4}

Experiments 3 and 4 examined the effects of varying the number of frames for 50 - and $500-\mathrm{msec}$ SOAs.

\section{Method}

Displays were presented with frame-to-frame angular displacements of $6^{\circ}, 18^{\circ}$, or $30^{\circ}$ and with sequences of two, three, four, six, or nine frames. Each possible combination of angular displacement and number of frames occurred five times during each block of trials. Every display contained 4 line segments and the individual frames were presented with a 0 -msec ISI. SOAs were $50 \mathrm{msec}$ in Experiment 3, and $500 \mathrm{msec}$ in Experiment 4.

\section{Results}

The data from Experiment 3, with 50-msec SOAs, are presented in Figure 5. An analysis of variance revealed that there were large effects of angular displacement $[F(2,6)=56.91, p<.001]$ and number of frames

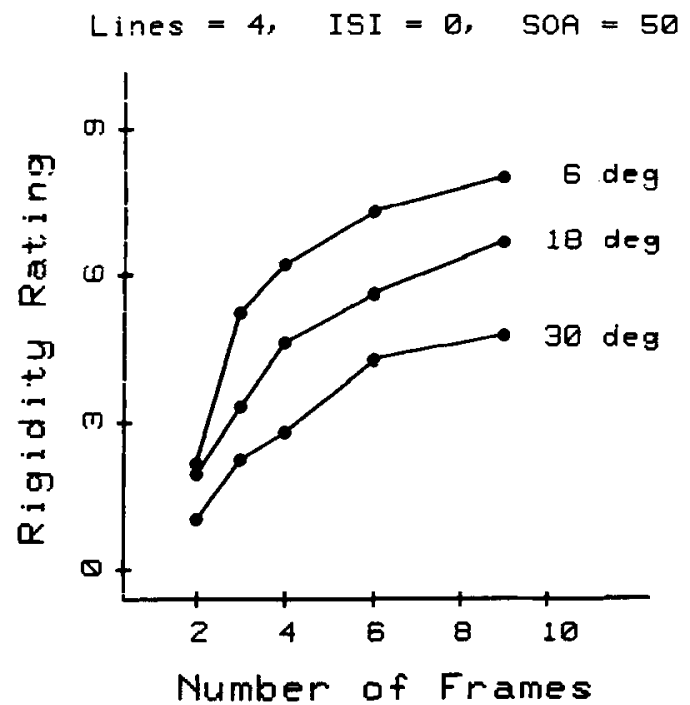

Figure 5. Mean rigidity ratings of 4 observers as a function of the number of frames for the 50-msec-SOA sequences in Experiment 3, with $6^{\circ}, 18^{\circ}$, and $30^{\circ}$ angular displacements.

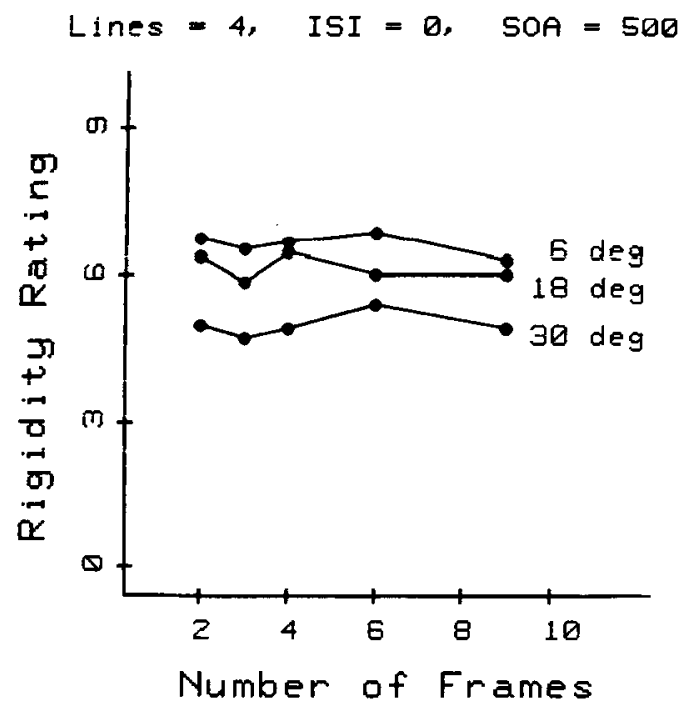

Figure 6. Mean rigidity ratings of 4 observers as a function of the number of frames for the 500 -msec-SOA sequences in Experiment 4, with $6^{\circ}, 18^{\circ}$, and $30^{\circ}$ angular displacements.

$[F(4,12)=61.12, p<.001]$. In Experiment 4, with 500msec SOAs, there was a much smaller effect of angular displacement $[F(2,6)=22.75, p<.001]$ and no effect at all of number of frames $[F(4,12)=1.83, p>.05]$ (see Figure 6).

To summarize briefly, our results thus far indicate that there are at least two different sets of conditions in which it is possible to perceive the rigid rotation in depth of a wire-frame figure. One set of conditions includes multipleframe sequences with small angular displacements and small SOAs. The other includes large SOAs and small displacements, with no restrictions on the number of frames. As a result of these observations, all stimulus manipulations in Experiments 5-9 were considered separately in two different contexts-with 500-msec SOAs in two-frame sequences, and with 50-msec SOAs in nineframe sequences.

\section{EXPERIMENT 5}

Experiment 5 was designed to examine the effects of varying the ISI for two-frame sequences. Nine-frame sequences could not be investigated with varying ISIs because of the limitations of our apparatus.

\section{Method}

Displays were presented with angular displacements of $6^{\circ}, 18^{\circ}$, or $30^{\circ}$. Each display contained only two distinct frames depicting 4 line segments, with an SOA of $533 \mathrm{msec}$. The ISIs varied from one trial to the next, with possible values of $0,67,133,267$, and $467 \mathrm{msec}$. Each combination of angular displacement and ISI occurred five times during each block of trials.

\section{Results}

The results are presented in Figure 7. As is evident from the figure, there was a strong interaction between ISI and angular displacement. With $30^{\circ}$ angular displacements, 


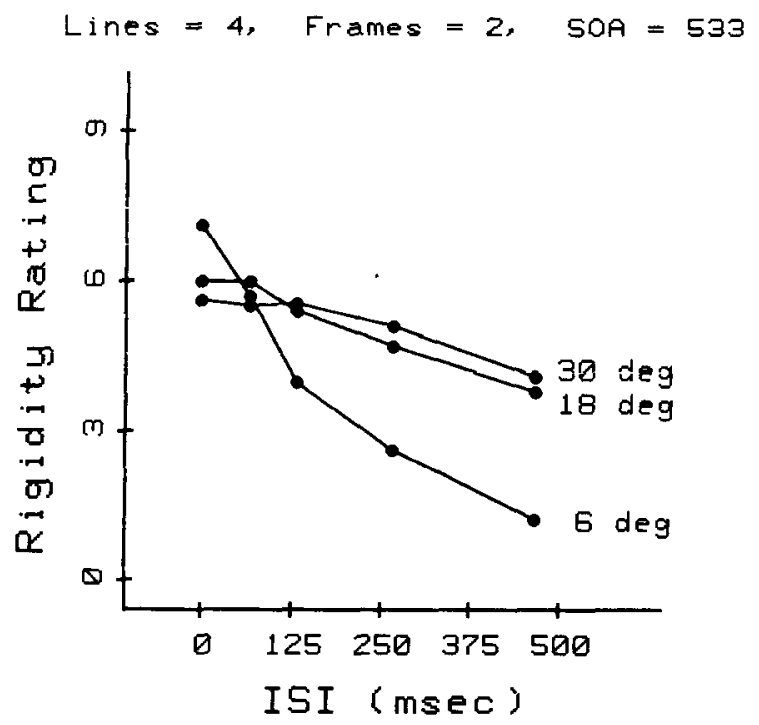

Figure 7. Mean rigidity ratings of $4 \mathrm{observers} \mathrm{as} \mathrm{a} \mathrm{function} \mathrm{of} \mathrm{ISI}$ for the two-frame, 533-msec-SOA sequences in Experiment 5, with $6^{\circ}, 18^{\circ}, 30^{\circ}$ angular displacements.

increasing the ISI from 0 to $467 \mathrm{msec}$ had a negligible effect on the observers' rigidity ratings $[F(4,12)=2.33$, $p>.05$ ], but with $6^{\circ}$ displacements the effect of increasing the ISI was dramatic $[F(4,12)=42.25, p<.001]$. Indeed, when the displays were generated with a $467-\mathrm{msec}$ ISI and a $6^{\circ}$ angular displacement, the depicted line segments appeared to be blinking on and off without moving at all. It is interesting to note, in this regard, that the condition using large ISIs was the only condition in all of our experiments in which a large angular displacement produced a more compelling impression of rigid rotation than could be achieved under comparable conditions with a smaller angular displacement.

\section{EXPERIMENTS 6 AND 7}

Experiments 6 and 7 examined the effects of varying the number of line segments with optimal timing for nineand two-frame motion sequences.

\section{Method}

Displays were presented with $4,8,16,21$, or 64 line segments; with angular displacements of $6^{\circ}, 18^{\circ}$, or $30^{\circ}$; and with a 0 -msec ISI. Each combination of angular displacement and number of lines occurred five times during each block of trials. Nine-frame sequences with 50-msec SOAs were employed in Experiment 6, and two-frame sequences with 500-msec SOAs were employed in Experiment 7 .

\section{Results}

The results from the nine-frame sequences are presented in Figure 8. An analysis of variance of these data revealed that there was a large effect of angular displacement $[F(2,6)=51.08, p<.001]$ (as in all of our previous experiments using similar parameters), but that there was no effect of number of lines $[F(4,12)=.58, p>.1]$.
The latter finding is also consistent with previous research reported by Green (1961) and Braunstein (1962), who used continuous motion displays.

The two-frame sequences again produced a pattern of results different from that of the nine-frame sequences, in that there was a strong interaction between angular displacement and number of lines (see Figure 9). With $30^{\circ}$ angular displacements, increasing the number of line segments from 4 to 64 significantly reduced the perceived rigidity of the displays $[F(4,12)=36.23, p<.001]$, whereas with $6^{\circ}$ displacements, a comparable increase in the number of line segments had no detectable effect $[F(4,12)=.44, p>.1]$.

\section{EXPERIMENTS 8 AND 9}

In addition to the effects of quantity of structure examined in Experiments 6 and 7, there are potential effects involving quality of structure that remain to be considered. For example, many of the computational models for perceiving structure from motion that have been proposed in the literature are exclusively concerned with the optical motions of identifiable points (see Todd, 1985). In applying such models to the displays used in the present experiments, only the endpoints of the line segments would be relevant. One might predict, therefore, that the perception of rigid rotation should be unaffected by presenting the endpoints in isolation. Experiments 8 and 9 were designed to test this prediction, using optimal timing parameters with nine- and two-frame motion sequences.

\section{Method}

Displays containing 4 line segments were presented with a 0 -msec ISI and with angular displacements of $6^{\circ}, 18^{\circ}$, or $30^{\circ}$. Each segment of a figure was displayed as a linear configuration of dots

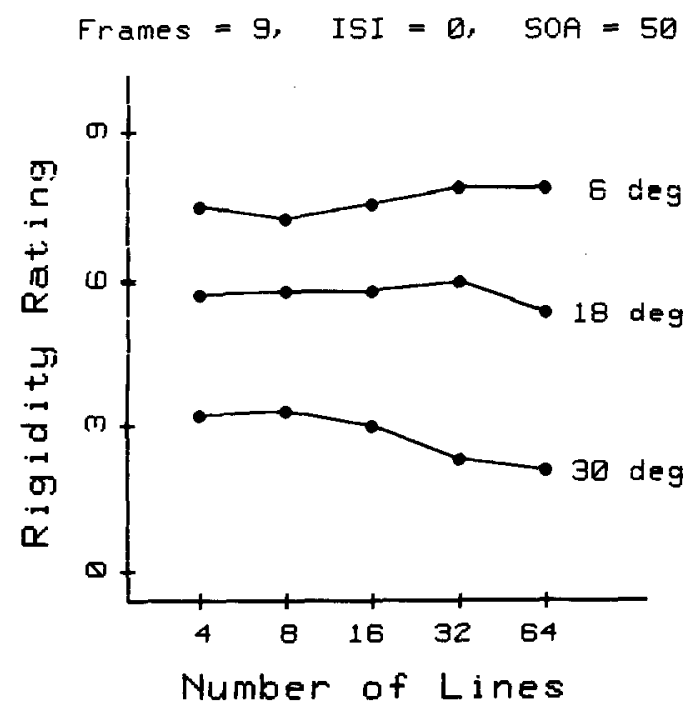

Figure 8. Mean rigidity ratings of 4 observers as a function of the number of lines for the nine-frame, 50-msec-SOA sequences in Experiment 6 , with $6^{\circ}, 18^{\circ}$, and $30^{\circ}$ angular displacements. 


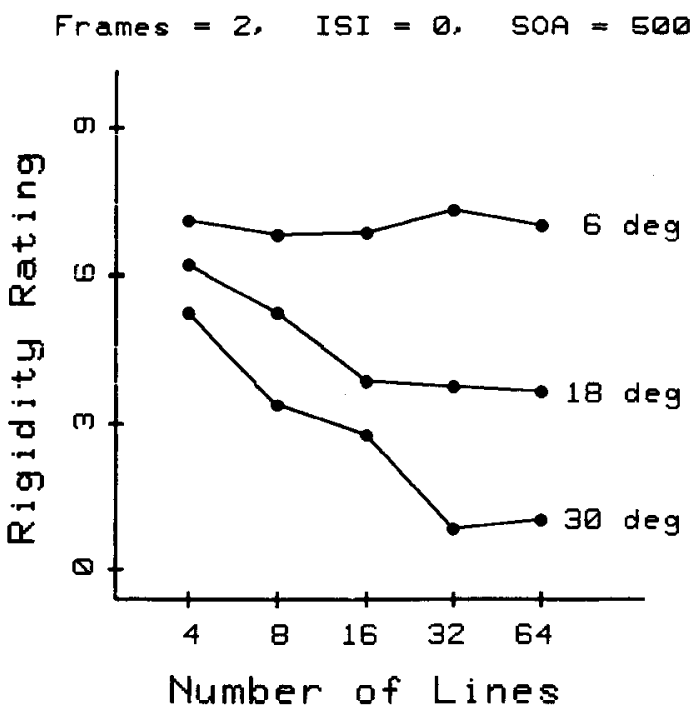

Figure 9. Mean rigidity ratings of 4 observers as a function of the number of lines for the two-frame, 500-msec-SOA sequences in Experiment 7 , with $6^{\circ}, 18^{\circ}$, and $30^{\circ}$ angular displacements.

(e.g., see Figure 1), rather than as a solid line as in previous experiments. A segment could be defined with $1,2,4,8$, or 16 dots. One dot was always positioned at the endpoint most peripheral to the origin, and additional dots, when present, were positioned at equal intervals toward the opposite endpoint. Each possible combination of angular displacement and number of dots occurred five times during each block of trials. Nine-frame sequences with 50msec SOAs were employed in Experiment 8, and two-frame sequences with 500-msec SOAs were employed in Experiment 9.

\section{Results}

The results for the nine- and two-frame conditions are presented in Figures 10 and 11, respectively. An analysis of variance revealed that in each case the observers' ratings of rigidity were significantly reduced when the number of dots per line dropped below $4[F(4,12)=$ $32.89, p<.001$, for the nine-frame sequences, and $F(4,12)=29.88, p<.001$, for the two-frame sequences]. When the number of dots per line was $\geq 4$, the ratings were equivalent to those obtained with continuous contours. A similar difference in perceived rigidity for configurations of dots and lines was reported by Green (1961) for continuous motion displays. Taken together, these findings suggest that 4 dots define a line for the perception of rigid rotation, and that the projectively changing length and orientation of a line provides perceptually useful information that is not available from the motion of its endpoints presented in isolation (see also Todd, 1985).

It is interesting to compare these results with the finding in Experiment 7 that rigidity ratings for two-frame sequences can be significantly reduced by increasing the number of moving elements. Note in Figure 11 that the opposite effect was produced in Experiment 9-that is to say, increasing the number of elements produced a cor- responding increase in the perceived rigidity of the displays. The difference, we believe, is that the individual dots in Experiment 9 were organized into a small number of higher order colinear units, thus reducing their information load. It is not the number of identifiable points per se that affects perceived rigidity in two-frame sequences, but the overall information load of the entire pattern (cf. Shepard \& Judd, 1976).

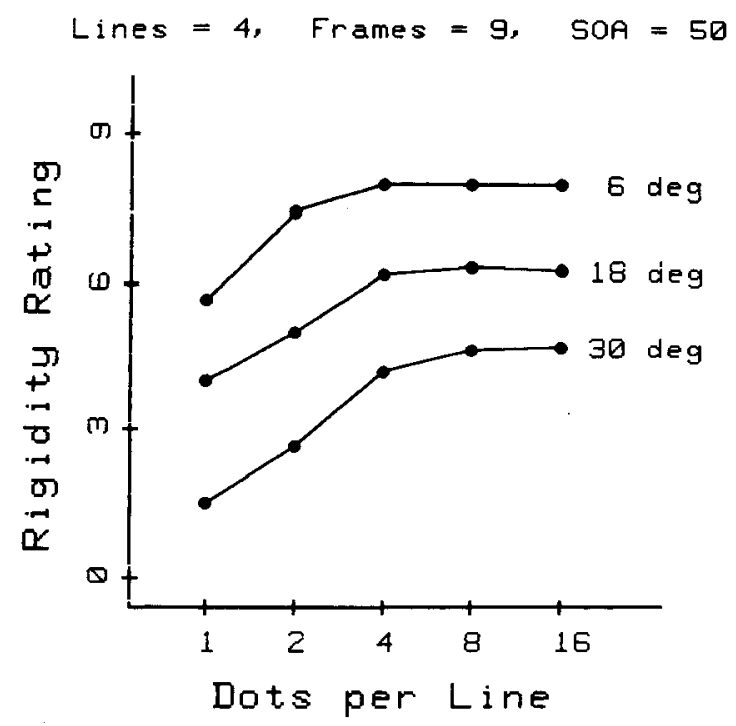

Figure 10. Mean rigidity ratings of 4 observers as a function of the number of dots per line for the nine-frame, 50-msec-SOA sequences in Experiment 8, with $6^{\circ}, 18^{\circ}$, and $30^{\circ}$ angular displacements.

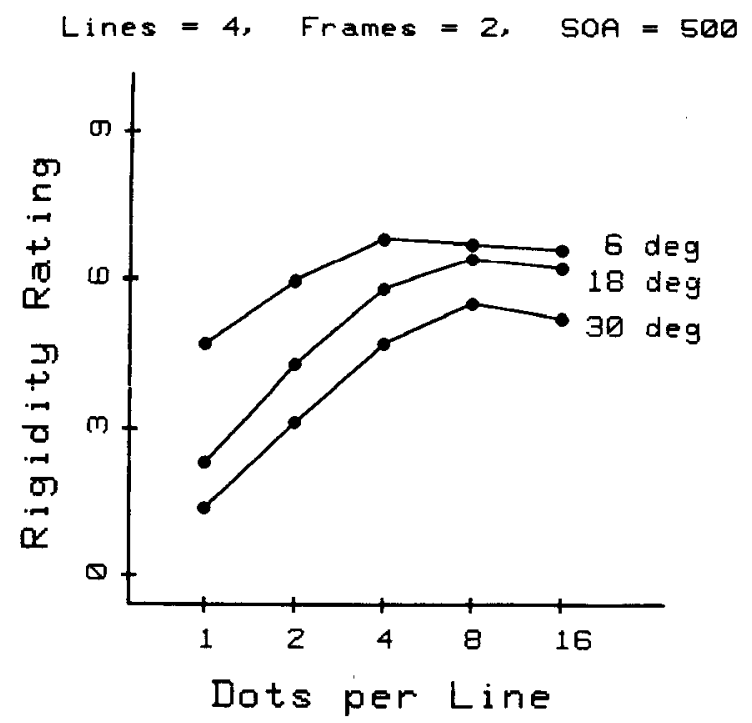

Figure 11. Mean rigidity ratings of 4 observers as a function of the number of dots per line for the two-frame, 500-msec-SOA sequences in Experiment 9, with $6^{\circ}, 18^{\circ}, 30^{\circ}$ angular displacements. 


\section{EXPERIMENTS 10 AND 11}

Another important aspect of structural quality that could potentially affect an observer's perception of rigid motion is the presence of an optical flow field. Flow fields arise when opaque surfaces are viewed; they are characterized by smooth variations over space in the visual motions of neighboring optical elements. Recent theoretical analyses by Koenderink and van Doorn $(1975,1977)$ and others have demonstrated that smooth optical flow fields provide potential information about an object's 3-D form that is not available from the optical distortions of other types of structural configurations (e.g., the wire-frame figures used in the present experiments). If there are specialized mechanisms within the human visual system for the analysis of this information, then it is quite likely that their spatiotemporal sensitivities could be different from those of the mechanisms employed in other contexts. Experiments 10 and 11 were designed, therefore, in an effort to determine whether the presence of a continuous flow field can alter the acceptable timing parameters for the perception of rigid rotation.

\section{Method}

The displays were generated using computer simulations of a vertically oriented cylinder, with a length of 220 pixels $(22 \mathrm{~cm})$ and a diameter of 100 pixels $(10 \mathrm{~cm})$, rotating in depth under parallel projection with a frame-to-frame angular displacement of $6^{\circ}$ and a 0-msec ISI. There were three separate conditions. In the opaque surface condition, 1,000 points were positioned at random on the surface of the simulated cylinder, but only those points on the front half of the surface were visible in each frame of the motion sequence (i.e., the back half of the surface was occluded). In the transparent surface condition, 500 points were positioned at random on the surface of the cylinder and all of the points were visible in every frame. In the transparent volume condition, 500 points were again visible in every frame, but were positioned at random within the volume of the cylinder.

It is important to note that only the opaque surface condition produced smooth variations over space in the visual motions of neighboring optical elements, which is required to achieve a continuous flow field. In the transparent surface condition, the projected displacements of neighboring elements were always similar in magnitude, but could be oriented in opposite directions. In the transparent volume condition, the projected displacements of neighboring elements could vary discontinuously in both magnitude and direction.

Displays were presented with possible SOAs of $37.5,50,100$, 200 , and $400 \mathrm{msec}$, each of which occurred five times for each condition during every block of trials. Nine-frame sequences were employed in Experiment 10, and two-frame sequences were employed in Experiment 11. Three of the authors (J.T., R.A., and F.R.) participated in four blocks of trials for each experiment. As in the previous experiments, the initial block of trials was excluded from subsequent analyses.

\section{Results}

The results for the nine-frame sequences are presented in Figure 12. Note that the observers' ratings tended to decrease with increasing SOA $[F(4,8)=16.14$, $p<.001$ ] (see also Petersik, 1980). However, when the

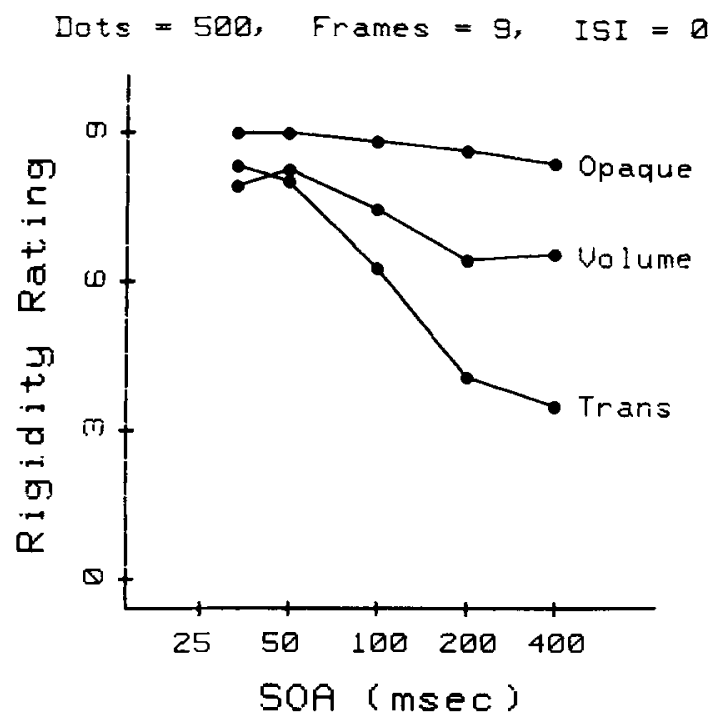

Figure 12. Mean rigidity ratings of 3 observers as a function of SOA for the nine-frame sequences in Experiment 10 depicting an opaque cylindrical surface, a transparent cylindrical surface, and a filled cylindrical volume.

overall pattern of optical motion formed a smooth flow field (i.e., in the opaque surface condition), the perception of rigid rotation could tolerate significantly longer SOAs than it could when the necessary conditions for a flow field were violated $[F(1,4)=50.16, p<.001]$. A similar difference between transparent and opaque surfaces was reported by Braunstein and Andersen (1984), who used judgments of perceived shape rather than rigidity.

For the two-frame sequences, it was the shorter SOAs for which perception of rigid rotation was most difficult $[F(4,8)=54.41, p<.001]$, but the overall pattern of ratings among the three structural configurations was equivalent to the pattern obtained for the nine-frame sequences (see Figure 13). That is to say, the opaque surface condition produced higher ratings than did the other conditions, which did not produce smooth flow fields $[F(1,4)=97.01, p<.001]$.

It is interesting to compare these results from the twoframe sequences with those obtained in an earlier series of experiments by Doner et al. (1984). These authors examined the ability of observers to discriminate the relative coherence of rotating spherical surfaces under a variety of different stimulus conditions. In one particularly relevant experiment, they compared observers' discrimination accuracy for two-frame apparent motion sequences with varying SOAs of 11 and $45 \mathrm{msec}$. Performance was quite high with the 45-msec SOAs, but was no greater than chance when the SOAs were reduced to $11 \mathrm{msec}$. These results are similar to those we obtained with twoframe sequences of opaque surfaces in Experiment 11. It is important to note, however, that the displays used by Doner et al. were designed to simulate transparent, 


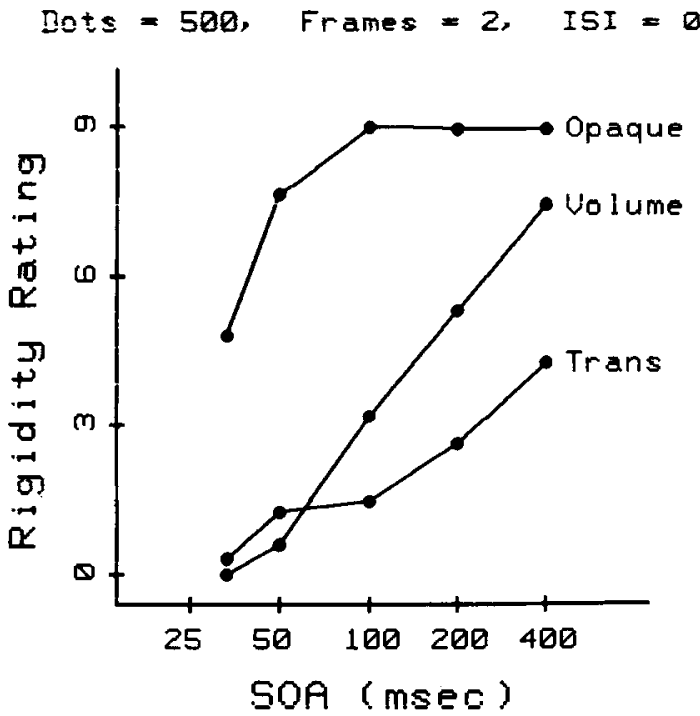

Figure 13. Mean rigidity ratings of 3 observers as a function of SOA for the two-frame sequences in Experiment 11 depicting an opaque cylindrical surface, a transparent cylindrical surface, and a filled cylindrical volume.

rather than opaque surfaces. Thus it would appear that their results stand in sharp contrast to those shown in Figure 13. Indeed, when two-frame sequences of a transparent cylinder were presented in the present experiments, the perception of rigid rotation could not be reliably elicited at any SOA.

Doner et al. (1984) noted that the perception of coherent motion in their displays required the use of an exaggerated polar perspective (as opposed to the parallel perspective used in the present experiments). We have confirmed this finding in our own laboratory. In doing so, however, we noted a curious phenomenon-namely, that the front half of the surface under these conditions appears to be invisible. This phenomenon arises because of the exaggerated polar perspective used to generate the displays. When a transparent sphere (or cylinder) is viewed under parallel perspective, the projected elements from the front and back half of its surface move in opposite directions. As viewing distance becomes smaller and smaller, the relative balance of these projected displacements becomes significantly skewed. When the viewing distance is one spherical diameter from its center (i.e., the conditions used by Doner et al.), the elements contained within the rearmost three quarters of the surface all move projectively in the same direction. In other words, the exaggerated polar perspective approximates the uniform pattern of optical flow created by a concave opaque surface. If the displays used by Doner et al. are considered as opaque surfaces, as is suggested by this analysis, then their results are generally consistent with those obtained in the present investigation.

\section{GENERAL DISCUSSION}

The present series of experiments was designed to explore the overall range of conditions in which it is possible to perceive apparent motion of a rigid object rotating in depth. Several different display parameters were manipulated, including the number of frames in the motion sequence; the SOA, ISI, and angular displacement between frames; the number of elements in the depicted object; and the structural organization of those elements. The results indicate that there are four distinct combinations of parameters that are capable of eliciting a perceptually compelling impression of apparent motion in depth, each of which is described briefly below.

Condition 1. The best possible condition for the perception of rigid rotation employs multiple-frame sequences with small angular displacements and small SOAs (see Experiments 1, 3, 6, 8, and 10). Moving configurations of connected lines appear to work better than configurations of isolated points (see Experiment 8; Green, 1961), but the number of points or lines has no discernible effect on observers' rigidity ratings (see Experiments 6 and 10; Green, 1961; Braunstein, 1962).

Condition 2. The next best condition for the perception of rigid rotation employs smooth flow fields with small displacements and small ISIs. Although ISI and displacement were not manipulated with flow-field stimuli in the present experiments, their effects can be inferred from the findings of previous investigations (e.g., Bell \& Lappin, 1973; Braddick, 1974; Doner et al., 1984; Lappin \& Bell, 1976). The present study showed that the perception of rigid rotation can be achieved with smooth flow fields over a wide range of SOAs, regardless of the number of frames in a sequence (see Experiments 10 and 11). The amount of structure required to define a perceptually adequate flow field has yet to be determined, but there is considerable evidence to indicate that the perceptual analysis of this type of stimulus can tolerate large amounts of noise (Doner et al., 1984; Lappin et al., 1980; Todd, 1985; van Doorn \& Koenderink, 1982a, 1982b, 1982c).

Condition 3. A third, although somewhat less compelling, condition in which it is possible to perceive rigid rotation employs large SOAs, small angular displacements, and small ISIs (see Experiments 2, 4, 7, 9, and 11). Observers' rigidity ratings under these conditions were higher when displays were composed of connected line segments, rather than isolated dots (see Experiment 9). The ratings were unaffected, however, by the number of frames in a sequence (see Experiment 4) or the number of elements depicted in each frame (see Experiments 7 and 11).

Condition 4. The least effective combination of parameters used in our investigation that still permitted the perception of rigid rotation included large SOAs, large angular displacements, and spatial configurations of 
limited structural complexity (see Experiments 2, 4, 5, 7 , and 9). Observers' rigidity ratings under these conditions were unaffected by large variations in ISI (see Experiment 5) or the number of frames in a sequence (see Experiment 4). The difficulty of perceiving rigid rotation with large angular displacements of structurally complex displays was revealed in Experiment 7 (see also Doner et al, 1984). Structural complexity in this context is influenced by the number of moving elements in a display, but it is also affected by how those elements are organized (see, e.g., Experiment 9; Shepard \& Judd, 1976).

It is interesting to note that the perception of rigid rotation could tolerate small SOAs in only two of the conditions described above-namely, with multiple-frame sequences in Condition 1 or with smooth flow fields in Condition 2 . These were also the only two conditions in which the impression of rigid rotation could be achieved without cognitive effort from the onset of the motion sequence. Both of these conditions seem to involve a smooth integration of visual information from moving optical elements. This integration apparently can occur either over space, with smooth flow fields, or over time, with multiple-frame sequences.

With two-frame sequences that did not form smooth flow fields, the perception of rigid rotation could be achieved only with large SOAs of several hundred milliseconds (i.e., when Condition 3 or Condition 4 was satisfied) and with at least some degree of cognitive effort on the part of the observer. Under these conditions, the impression of rigid rotation often required several seconds of viewing in which there was a conscious attempt to mentally rotate the elements or to mentally invert some of them in depth so that they would all appear to rotate in the same direction. This suggests that the perception of rigid rotation from two-frame sequences may be critically dependent on a repetitive oscillation of the displays.

\section{THEORETICAL CONSIDERATIONS}

Although the study of apparent motion has a long history within perceptual psychology, it has been largely restricted to two-frame sequences depicting simple displacements in 2-D space. It is now generally accepted that there are two distinct combinations of display parameters that are capable of producing a perceptually compelling impression of 2-D motion (e.g., see Bell \& Lappin, 1973; Braddick, 1974; Kolers, 1972; Lappin \& Bell, 1976). One set of parameters permits uniform translations or rotations of densely structured patterns, but is restricted to monocularly presented sequences with small spatial displacements, small ISIs, and adequate amounts of luminance contrast. The other permits binocularly presented sequences with isoluminant chromatic contours over a wide range of ISIs and spatial displacements, but is restricted to patterns of limited structural complexity. On the basis of these findings, Braddick (1974) argued that there are two distinct mechanisms for the detection of optical displacements, which he has referred to as the short-range and long-range processes.

Could multiple mechanisms of motion perception have been at work in the present experiments? Perhaps for Conditions 1 and 2 this is a reasonable hypothesis. Recent theoretical analyses have shown that at least two kinds of information are provided by optical motion. One is based on the trajectories of optical elements over an extended interval of time (e.g., Todd, 1982; Ullman, 1984), whereas the other is based on the relative motions within a neighborhood of optical elements at an instantaneous moment in time (e.g., Koenderink, 1986; Koenderink \& van Doorn, 1975, 1977). It is reasonable to suppose that our visual systems could have evolved specialized mechanisms for the analysis of these different types of information. One of these mechanisms would presumably be sensitive to gradual changes in individual elements, as revealed over several frames in an apparent motion sequence, whereas the other would be driven by the relative displacements of neighboring elements at each frame transition. We would expect there to be some physical limitations of these mechanisms that would constrain the ranges of space and time over which information could be successfully integrated. This would determine the perceptually acceptable displacements, frame rates, and so forth, of an apparent motion sequence.

Other limitations on an observer's ability to perceive rigid motion are based on the information potentially available in a display, and are independent of any physical constraints on the visual system. These limitations arise, for example, when the pattern of optical motion in a display has no mathematically possible rigid interpretation (e.g., see Todd, 1982). It is important to keep in mind that all of the displays employed in our experiments were designed to simulate an actual object rotating rigidly in 3-D space under parallel projection. Not all of the displays, however, had unique rigid interpretations. When a motion sequence was reduced to only two frames, there were multiple rigid interpretations to which it could projectively correspond (Ullman, 1979).

It remains to be demonstrated whether the visual motion in these displays contains perceptually useful information about an object's 3-D form. The present research was intended as a first step toward addressing this issue. Although rigidity ratings are an admittedly crude psychophysical procedure, they are a convenient tool for quickly mapping the optimal timing parameters for the perception of rigid rotation under a wide variety of theoretically relevant conditions. One of the implications of this research, for example, is that two-frame sequences require longer SOAs than are appropriate for multiple-frame sequences (see Sperling, 1976). Using the parameters shown by the present study to be optimal for different conditions, we are currently performing a follow-up investigation in an effort to measure observers' sensitivities to 
the precise 3-D structures of wire-frame figures rotating in depth. The results of these experiments will be reported in a later article.

\section{REFERENCES}

BeLl, H. H., \& LAPPIN, J. S. (1973). Sufficient conditions for the discrimination of motion. Perception \& Psychophysics, 14, 45-50.

Braddick, O. (1974). A short-range process in apparent motion. $V i$ sion Research, 14, 519-527.

Braunstein, M. L. (1962). Depth perception in rotating dot patterns: Effects of numerosity and perspective. Journal of Experimental Psychology, 64, 415-420.

Braunstein, M. L., \& ANDersen, G. J. (1984). Shape and depth perception from parallel projections of three-dimensional motion. Journal of Experimental Psychology: Human Perception \& Performance, 10, 749-760.

Braunstein, M. L., Hoffman, D. D., Shapiro, L. R., Andersen, G. J., BENNETT, B. M. (1987). Minimum points and views for recovery of three-dimensional structure. Journal of Experimental Psychology: Human Perception \& Performance, 13, 335-343.

Clocksin, W. F. (1980). Perception of surface slant and edge labels from optical flow: A computational approach. Perception, 9, 252-269.

Doner, J., Lappin, J. S., \& Perfetro, G. (1984). Detection of threedimensional structure in moving optical patterns. Journal of Experimental Psychology: Human Perception \& Performance, 10, 1-11.

GREEN, B. F. (1961). Figural coherence in the kinetic depth effect. Journal of Experimental Psychology, 62, 272-282.

HoffMAn, D., \& BENNETT, B. (1985). Inferring the relative 3-D positions of two moving points. Journal of the Optical Society of America, 75, 350-533.

HoffMAN, D., \& BenNetT, B. (1986). The computation of structure from fixed axis motion: Rigid structures. Biological Cybernetics, 54, $1-13$.

KoenderInK, J. J. (1986). Optic flow. Vision Research, 26, 161-180.

KoEnderink, J. J., \& VAN DoORN, A. J. (1975). Invariant properties of the motion parallax field due to the motion of rigid bodies relative to the observer. Optica Acta, 22, 773-791.

KoENDERINK, J. J., \& VAN DOORN, A. J. (1977). How an ambulant observer can construct a model of the environment from the geometrical structure of visual flow. In G. Hauske \& E. Butenandt (Eds.), Kybernetik (pp. 225-247). Munich: Oldenberg.

Kolers, P. A. (1972). Aspects of motion perception. Oxford: Pergamon Press.

LAPPIN, J. S., \& BELL, H. H. (1976). The detection of coherence in moving random dot patterns. Vision Research, 16, 161-168.

LAPPIN, J. S., Doner, J. F., \& KotTas, B. (1980). Minimal conditions for the visual detection of structure and motion in three dimensions. Science, 209, 717-719.

LAPPIN, J. S., \& FuQUA, M. A. (1983). Accurate visual measurement of three-dimensional moving patterns. Science, 221, 480-482.
LEE, D. N. (1974). Visual information during locomotion. In R. B. McLeod \& H. Pick (Eds.), Perception: Essays in honor of James Gibson (pp. 250-267). Ithaca, NY: Cornell University Press.

Longuet-Higgens, H. C., \& Prazdny, K. (1980). The interpretation of a moving retinal image. Proceedings of the Royal Society of London, 208, 385-397.

Nakayama, K., \&oomis, J. M. (1974). Optical velocity patterns, velocity sensitive neurons, and space perception: A hypothesis. Perception, 3, 53-80.

Petersik, J. T. (1980). The effects of spatial and temporal factors on the perception of stroboscopic motion simulations. Perception, 9 , 271-283.

Prazdny, K. (1980). Egomotion and relative depth map from optical flow. Biological Cybernetics, 36, 87-102.

SHEPARD, R. N., \& JUDD, S. A. (1976). Perceptual illusion of rotation of three-dimensional objects. Science, 191, 952-954.

SPERLING, G. (1976). Movement perception in computer-driven visual displays. Behavior Research Methods \& Instrumentation, 8, 144-151.

ToDD, J. T. (1981). Visual information about moving objects. Journal of Experimental Psychology: Human Perception \& Performance, 7 , 795-810.

TonD, J. T. (1982). Visual information about rigid and nonrigid motion: A geometric analysis. Journal of Experimental Psychology: $\mathrm{Hu}$ man Perception \& Performance, 8, 238-252.

ToDD, J. T. (1984). The perception of three-dimensional structure from rigid and nonrigid motion. Perception \& Psychophysics, 36, 97-103.

ToDD, J. T. (1985). Perception of structure from motion: Is projective correspondence of moving elements a necessary condition? Journal of Experimental Psychology: Human Perception \& Performance, 11, 689-710.

UlLMaN, S. (1979). The interpretation of visual motion. Cambridge, MA: MIT Press.

UlLman, S. (1984). Maximizing rigidity: The incremental recovery of 3-D structure from rigid and nonrigid motion. Perception, 13, 255-274.

van DoOrn, A. J., \& Koenderink, J. J. (1982a). Spatial properties of the visual detectability of moving spatial white noise. Experimental Brain Research, 45, 189-195.

VAN DoORn, A. J., \& KoEnderink, J. J. (1982b). Temporal properties of the visual detectability of moving spatial white noise. Experimental Brain Research, 45, 179-188.

VAN DOORN, A. J., \& KOENDERINK, J. J. (1982c). Visibility of movement gradients. Biological Cybernetics, 44, 167-175.

WaLlach, H. , \& O'CONNELL, D. N. (1953). The kinetic depth effect. Journal of Experimental Psychology, 45, 205-217.

WebB, J. A., \& AGGarWAL, J. K. (1981). Visually interpreting the motion of objects in space. Computer, 8, 40-46.

(Manuscript received May 26, 1987; revision accepted for publication August $25,1987$. 